\section{Organic Chemistry: From Fundamentals to Advance Chemical Research}

\author{
Rajesh H Tale \\ SRTM University, Nanded, Maharashtra, \\ India
}

Received: June 27, 2016; Accepted: June 28, 2016; Published: June 30, 2016
As is the well-established fact that the chemistry is multiconnected and core science among the various pure science disciplines. Among various branches of chemistry itself, undoubtly, organic chemistry is key subarea. The very essence of life, the cell, to major extent itself lies within a domain of organic chemistry. It may be from simple small molecules to the complex macromolecules, the organic chemistry is at work everywhere including drugs discovery, sustainable energy, green chemistry, agricultural productivity, electronics and the economy. Thus, organic chemistry research is considered to be the key to the success of the development of technology and in turn from the basis for the sustainable development of broad range of industries.

In a broader sense, current trend in organic chemistry research is mainly focused on two main areas. Firstly, the drug discovery as the continuously acquired bacterial resistance to the existence drugs make it mandatory to search for new generation drugs. Secondly, the material science, as the increasing demand due to the improved life standard and further expectations for sophisticated materials with hitherto inaccessible properties, durability, and safety have much sought after.

The many advancement in the field of material science seems to be much connected to the very fundamental principles of chemistry in general and organic chemistry in particular. It's not that the efforts have not been initiated in this direction and the concept is completely new. In the last decades, several discoveries based on the fundamental of chemistry have put forth to illustrate the theme. The following two examples worth mentioning here in order to have clean insight into the concept: 1) The concept of frustrated Lewis pairs (FLPs) and 2) H-bonding based suprmolecular assembly or supramolecular polymers.

It is the fundamental knowledge of chemistry that Lewis acids and Lewis bases usually form strong adduct and this behavior was described By Lewis [1]. However, it took almost century to disclose that intramolecular Lewis acid/Lewis base pair cleanly cleaves the dihydrogen molecule under ambient conditions. This discovery resulted into the new cooperative Lewis acid/base chemistry [2]. Such pairs are popularly known as frustrated Lewis pair(FLP) The further advancement in the field had remarkable consequence which lead to the development of FLP catalysts for the hydrogenation of a range of organic substrates [3].
Corresponding author: Rajesh H Tale

Đ rkht_2008@rediffmail.com

Associate Professor, SRTM University, Nanded, Maharashtra, India.

Citation: Tale RH. Organic Chemistry: From Fundamentals to Advance Chemical Research. J Org Inorg Chem. 2016, 2:1.

Another fundamental of chemistry that resulted into many paths breaking discovery is the $\mathrm{H}$-bonding. The role of $\mathrm{H}$-bonding is known for long time in the phenomena like DNA-base pairing, cage structure of water or dimerisation of small molecules (say acetic acid). The two popular polymers Kevlar_and Nylon 6, 6 originate from hydrogen bonding. In recent years, the supramolecular polymer chemistry (self assembly) has attracted much attention of chemist, in particular, polymer chemists as many properties of these materials can be tuned to the requirement through the judicious choise of $\mathrm{H}$-bond interactions and polymer structure Hydrogen bonding as a supramolecular handle due to advantages such as synthetic accessibility, directionality, fidelity, and, most importantly, their external stimuli responsiveness has been used extensively studied and well documented in the literature [4]. By designing suitable heterocyle based $\mathrm{H}$-bond donors, for instance, using ureidopyrimidinones, many new supramolecular polymers have been developed. These advance material find numerous applications (in liquid crystals) in the field of materials science.

In conclusion, it is clear that the potential of broad range of advanced materials appears to be almost limitless and hence there is large scope to grab these and many other fundamentals of organic chemistry or possibly inorganic chemistry to design the scheme of things for advance research in future. These future advancement would enhance further the already high impact of organic chemistry on scientific community in general and chemical community in particular. 


\section{References}

1 Lewis GN (1923) Valence and the Structure of Atoms and Molecules. Chemical Catalogue Company Inc., New York, USA.

2 Welch SGC, Juan RRS, Masuda JD, Stephan DW (2006) Reversible,
Metal-Free Hydrogen Activation. Science 314: 1124-1126.

3 Stephan DW, Erker Angew A (2015) Chem Int Ed 54: 2-44.

4 Yang SK, Zimmerman SC (2013) Hydrogen Bonding Modules for Use in Supramolecular Polymers. Isr J Chem 53: 511-520. 